\title{
Etude sur le revenu: la fin du commencement!
}

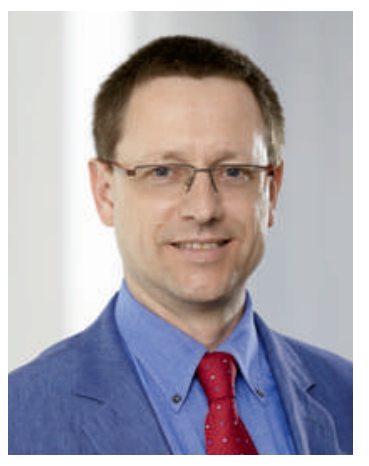

Pendant plus de 30 ans, le corps médical a opté pour la transparence en publiant sa propre étude sur le revenu des médecins. Dans un domaine d'activité financé en grande partie par l'argent de l'assurance sociale, l'intérêt public nous semblait important, et en dépit des questions récurrentes sur les données disponibles et sur leur interprétation, nous sommes restés longtemps attachés à la volonté de publier le revenu des médecins indépendants en exercice, à l'inverse d'autres groupes professionnels. Cette étude a été conçue par notre partenaire, le Bureau BASS, de manière indépendante en cherchant à optimiser la charge de travail et l'utilité de la collecte des données transmises par Medisuisse à la FMH.

Mais aujourd'hui, la situation juridique a changé car l'Office fédéral des assurances sociales ne délivre plus l'autorisation accordée initialement de transmettre les données. Il motive son choix par l'intérêt mineur que porte l'opinion publique à toute cette procédure. Ainsi, la FMH n'a plus qu'à se soumettre à cette décision.

Ce changement ne nous empêche pas d'utiliser les ressources ainsi libérées pour nous tourner vers d'autres questions fondamentales: connaissons-nous suffisamment la situation professionnelle de nos membres? Quels sont leurs taux aux organisations représentées à la Chambre médicale de lui communiquer leur avis et leurs questions afin de pouvoir adapter ses travaux à venir aux attentes exprimées.

Le monde professionnel dans lequel exercent les médecins est en mutation. A l'instar de leurs collègues masculins, les femmes médecins souhaitent pouvoir associer travail et famille. Aujourd'hui, nous devons faire face au défi que nous lancent les nouveaux modèles de vie en couple, aussi bien

\section{La mutation du monde professionnel des médecins soulève des questions fondamentales.}

pour répondre au manque de personnel qualifié, qui touche toute l'économie, que pour donner suite aux investissements consentis dans le domaine de la formation. La vie de famille doit conserver ses droits même si les deux partenaires travaillent. Pour ce faire, il faut leur offrir la possibilité d'exercer une activité à temps partiel. Or, il ne s'agit pas uniquement du phénomène de la féminisation de notre profession. En effet, les incitatifs visant à réguler notre profession ne permettront pas à eux seuls d'améliorer la densité médicale dans les zones périphériques si les possibilités de travail pour le ou la partenaire et les commodités telles que la garde des enfants n'existent pas dans ces régions.

\section{Il faut aujourd'hui procéder à un état des lieux détaillé avec des données adéquates pour justifier du nombre de médecins dont nous aurons besoin demain.}

d'activité? Que signifie temps partiel? Où se pratique le travail à temps partiel, à l'hôpital ou en cabinet, et dans quelles spécialisations? Quelles sont les structures dans lesquelles exercent nos membres? S'agit-il de cabinets individuels, de cabinets de groupe ou encore de réseaux de soins? Les médecins sont-ils principalement indépendants ou exercent-ils une activité salariée? Au niveau régional, comment se répartissent ces différents aspects? Voilà, en bref, quelques-unes des questions les plus importantes qui se posent. Mais la FMH veut en savoir davantage, c'est la raison pour laquelle elle a demandé
Pour cibler notre politique professionnelle et l'adapter aux attentes de nos membres, nous avons besoin d'une large palette de données, et donc de la collaboration de toutes nos organisations et sociétés - mais avant tout, nous comptons sur nos membres pour qu'ils nous livrent leur point de vue!

Je vous remercie d'ores et déjà pour vos précieux retours.

Dr Christoph Bosshard, membre du Comité central de la FMH, responsable du domaine Données, démographie et qualité 